\title{
miR-15b inhibits cancer-initiating cell phenotypes and chemoresistance of cisplatin by targeting TRIM14 in oral tongue squamous cell cancer
}

\author{
XIJUN WANG $^{1,2}$, HONGMEI GUO ${ }^{3}$, BANJAMIN YAO $^{4}$ and JULIA HELMS ${ }^{4}$ \\ ${ }^{1}$ Department of Prosthodontics, ${ }^{2}$ Key Laboratory of Oral Biomedicine of Shandong Province, and \\ ${ }^{3}$ Department of Periodontology, School of Stomatology, Shandong University, Jinan, Shandong 250012, P.R. China; \\ ${ }^{4}$ Department of Surgery, University of California Davis, Sacaramento, CA 95817, USA
}

Received May 6, 2016; Accepted June 17, 2016

DOI: 10.3892/or.2017.5532

\begin{abstract}
Oral tongue squamous cell carcinoma (TSCC) is one of the most lethal cancers within the oral cavity and its prognosis remains dismal due to the paucity of effective therapeutic targets. The formation of cancer-initiating cells (CICs) and epithelial-mesenchymal transition (EMT) are pivotal events involved in the dismal prognosis. They have been shown to be related to the resistance to cisplatin treatment. In the present study, we showed that TRIM14 induced formation of cancer-initiating cells and EMT in TSCC SCC25 cells. Its overexpression promoted cisplatin resistance in the SCC25 cells. We found that overexpression of miR-15b suppressed TRIM14 and inhibited CIC phenotypes in the SCC25 cells. Moreover, overexpression of miR-15b promoted mesenchymal-epithelial transition (MET) in the SCC25 cells and sensitized cisplatin-resistant SCC25 (SCC25-res) cells to cisplatin. Thus, we conclude that miR-15b inhibited cancer stem cell phenotypes and its restoration reversed the chemoresistance of cisplatin by targeting TRIM14 in TSCC. Elucidating the molecular mechanism of EMT and cancer stem cells in TSCC may further aid in the understanding of the pathogenesis and progression of the disease, and offer novel targets for the discovery of new drugs.
\end{abstract}

\section{Introduction}

Oral squamous cell carcinoma (OSCC) represents the sixth most common solid cancer worldwide and tongue squamous carcinoma (TSCC) is one of the major oral malignant tumor subtypes (1-3). TSCC has the worst prognosis for early stage disease compared with any other head and neck cancer

Correspondence to: Dr Hongmei Guo, Department of Periodontology, School of Stomatology, Shandong University, 44-1 Wenhuaxi Road, Jinan, Shandong 250012, P.R. China E-mail: guohongmeisdu@163.com

Key words: tongue squamous cell carcinoma, cancer-initiating cells, epithelial-mesenchymal transition, miR-15b, TRIM14 subsite (4). Despite treatment with the combination of surgery, radiotherapy and chemotherapy, which lead to significant morbidity, failure rates remain unacceptably high $(4,5)$. One of the reasons for the dismal prognosis is that current treatment cannot eliminate cancer-initiating cells (CICs) $(6,7)$. A comprehensive understanding of the molecular basis of CICs in TSCC may contribute to the identification of novel therapeutic targets to improve patient outcome.

Tripartite motif containing 14 (TRIM14), a newly identified gene located on chromosome 9q22, contains a B-box, a coiled-coil domain, and a C-terminal PRYSPRY domain, but lacks the N-terminal RING domain found in most TRIM family proteins (8). TRIM14 is markedly upregulated in TSCC cell lines and clinical tissues and its expression was found to be significantly correlated with the TNM classification in patients with TSCC (9). TRIM14 expression may be an independent prognostic indicator for the survival of patients with TSCC (9). Ectopic expression of TRIM14 in TSCC cells promoted proliferation, angiogenesis and increased resistance to cisplatin-induced apoptosis of TSCC cells (9). Furthermore, TRIM14 overexpression significantly promoted the tumorigenicity of TSCC cells in vivo whereas silencing of endogenous TRIM14 caused an opposite outcome (9). It has been proposed that TRIM14 may represent a novel therapeutic target for the treatment of TSCC (9).

MicroRNAs (miRNAs/miRs) are regulatory, non-coding RNAs $~ 18-25$ nucleotides in length and are expressed at specific stages of tissue development or cell differentiation, and have large-scale effects on the expression of a variety of genes at the post-transcriptional level (10-15). miRNAs induce mRNA degradation or translational suppression of targeted transcripts by base-pairing with the 3' untranslated region 3'UTR of its targeted mRNAs (10-15). Dysregulated miRNAs have an important role in the development of TSCC (16). However, elucidating the roles of miRNAs in cancer biology, particularly in TSCC, remains an ongoing process.

In the present study, we showed that TRIM14 induced the formation of CICs and epithelial-mesenchymal transition (EMT) in TSCC SCC25 cells. Its overexpression promoted cisplatin-resistance in the SCC25 cells. We found that overexpression of miR-15b suppressed TRIM14 and inhibited 
cancer-initiating cell phenotypes in the SCC25 cells. Moreover, overexpression of miR-15b promoted mesenchymal-epithelial transition (MET) in the SCC25 cells and sensitized cisplatinresistant SCC25 (SCC25-res) cells to cisplatin. Thus, we conclude that miR-15b inhibited cancer stem cell phenotypes and its restoration reversed chemoresistance of cisplatin by targeting TRIM14 in TSCC. Elucidating the molecular mechanism of EMT and cancer-initiating cells in TSCC may further help us to understand the pathogenesis and progression of the disease, and offer novel targets for the discovery of new drugs.

\section{Materials and methods}

Human TSCC cell line SCC25 and SCC25-res cells. SCC25 cells were purchased from the American Type Culture Collection (ATCC; Manassas, VA, USA). To obtain cisplatin-resistant tongue cancer cells, we treated SCC25 cells with escalating concentrations of cisplatin from $10^{7}$ to $10^{5} \mathrm{M}$. The established SCC25-res cells grew at a similar rate in the presence or absence of $10^{5} \mathrm{M}$ cisplatin for 3 days (data not shown). $\mathrm{IC}_{50}$ is the cisplatin concentration that reduces proliferating cells by $50 \%$. The $\mathrm{IC}_{50}$ value of SCC25-res cells increased by 12-fold, respectively, as compared with the SCC25 cells (data not shown).

Western blotting. Protein extracts were resolved through SDS-polyacrylamide gel electrophoresis, transferred to polyvinylidene difluoride membranes (Bio-Rad, Berkeley, CA, USA), and probed with antibodies against human ERCC1, YAP, TRIM14, CD44, CD133, OCT4, Nanog (all from Abcam, Cambridge, MA, UK) or $\beta$-actin (Santa Cruz Biotechnology, Santa Cruz, CA, USA).

Sphere forming assay. Cells were seeded on $0.5 \%$ agar precoated 6-well plates and cultured for 1 week. Single spheres were selected and counted.

Soft agar assay. The soft agar assay was carried out as previously described (17).

MTT assay. To monitor resistance to cisplatin, SCC25 and SCC25-res cells were treated with cisplatin at different concentrations for $24 \mathrm{~h}$. The MTT assay was performed as previously described (18).

Real-time PCR for miRNAs. Total RNA was isolated from cultured cells using TRIzol reagent (Invitrogen, Carlsbad, CA, USA). Detection of the mature form of miRNAs and the mRNA expression of TRIM14 were performed using specific qRT-PCR primers. The primer sequences of GAPDH were: forward, 5'-ATTCAACGGCACAGTCAAGG-3' and reverse, 5'-GCAGAAGGGGCGGAGATGA-3'. The primer sequences of TRIM14 were: forward, 5'-GCAGAGACAGAGCTAGAC TGTAAAGGT-3' and reverse, 5'-CCTGGTCACACAATTGA TATGGA-3'.

Immunofluorescence staining. The cells after treatment were fixed with $4 \%$ paraformaldehyde and permeabilized with $1 \%$ Triton $\mathrm{X}$. After blocking, the cells were incubated with the primary antibodies against TRIM14, E-cadherin or vimentin (Abcam) overnight at $4^{\circ} \mathrm{C}$. On the following day, the cells were incubated with the secondary antibodies and 4',6-diamidine-2'-phenylindole dihydrochloride (DAPI), and viewed under a fluorescence microscope.

Methods of bioinformatics. The analysis of potential miRNA target sites was carried out using the commonly used prediction algorithms miRanda (http://www.microrna.org/).

Statistical analysis. Data are presented as mean \pm SEM. Student's t-test (two-tailed) was used to compare two groups. $\mathrm{P}<0.05$ was considered to indicate a statistically significant difference.

\section{Results}

TRIM14 induces the formation of the CIC phenotypes in $S C C 25$ cells. In order to ascertain whether TRIM14 affects CIC traits in SCC25 cells, we performed a sphere forming assay to assess the capacity of CIC or CIC-like self renewal in SCC25 cells. We tested whether TRIM14-expressing plasmids could stably express TRIM14 protein in the SCC25 cells. The results showed that TRIM14 protein was significantly increased by the TRIM14-expressing plasmids in the cells (Fig. 1A). Sphere forming assay showed that TRIM14-overexpressing cells formed much larger spheres after 14 days of culture as compared with the control cells, indicating markedly increased CIC traits by TRIM14-expressing plasmids (Fig. 1B). CD44, CD133, OCT4 and Nanog are positively associated with stem cell-like characteristics in cancer (19-22). To identify whether TRIM14 can regulate CD44, CD133, OCT4 and Nanog protein expression, we performed western blotting of the SCC25 cells transfected with the TRIM14-expressing plasmids and empty vectors. The results showed that CD44, CD133, OCT4 and Nanog protein were upregulated in the SCC25 cells transfected with the TRIM14-expressing plasmids (Fig. 1C). To determine whether cells with CIC characteristics could have increased clonogenic ability, we performed clonogenic assay. We found that clonogenic ability was significantly increased in the SCC25 cells transfected with the TRIM14-expressing plasmids (Fig. 1D). In addition, the results from the cell morphology demonstrated that overexpression of TRIM14 induced EMT phenotype in the SCC25 cells (Fig. 1E). We also performed western blotting to detect E-cadherin (epithelial marker) and vimentin (mesenchymal marker) protein in the SCC25 cells transfected with the TRIM14-expressing plasmids and empty vectors. We found that E-cadherin protein was downregulated and vimentin was upregulated by TRIM14 in the SCC25 cells transfected with the TRIM14-expressing plasmids (Fig. 1F).

TRIM14 overexpression leads to cisplatin-resistance in TSCC. To identify whether TRIM14 can affect cisplatin resistance in SCC25 cells, we performed MTT assay. We overexpressed TRIM14 in cisplatin-sensitive SCC25 cells and its overexpression transformed the cisplatin-sensitive SCC25 cells to cisplantin-resistant cells (Fig. 2A), suggesting that its overexpression is involved in cisplatin resistance. High expression of ERCC1 and YAP are associated with cispatin-resistance 
A

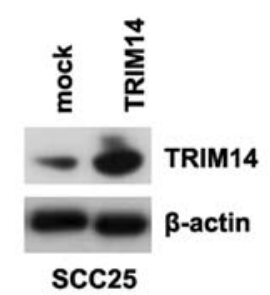

B
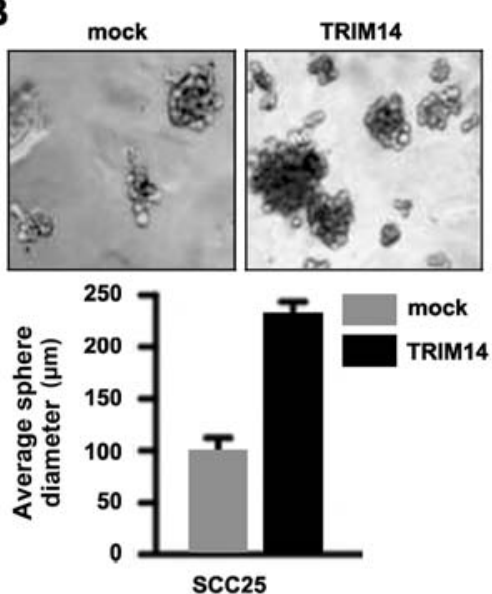

C

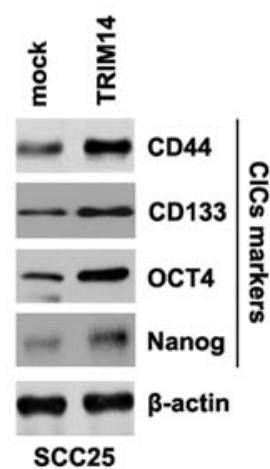

D

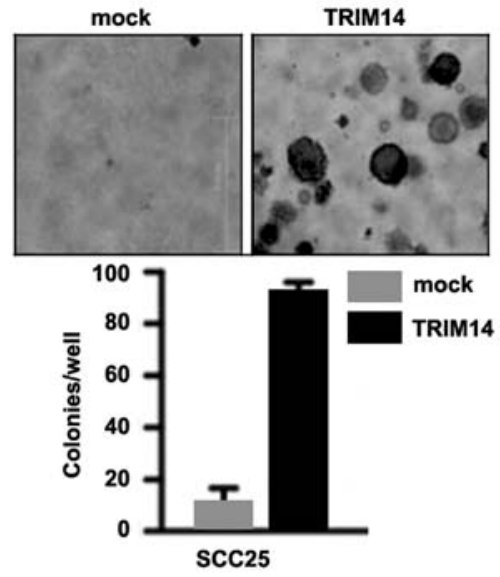

E

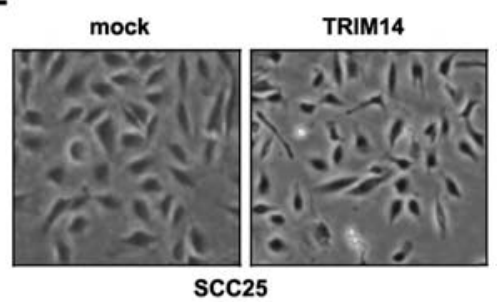

$\mathbf{F}$

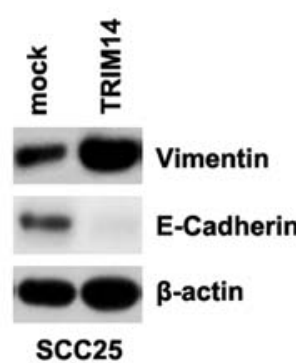

Figure 1. TRIM14 induces formation of cancer-initiating cell (CIC) phenotypes and epithelial-mesenchymal transition (EMT) in SCC25 cells. (A) Western blotting for TRIM14 in SCC25 cells transfected with TRIM14-expressing plasmids and empty vectors (mock). $\beta$-actin was used as a loading control; n=3. (B) Sphere growth of SCC25 cells transfected with TRIM14-expressing plasmids and empty vectors (mock). The data are reported as the average diameter of spheres formed/1,000 seeded cells. Upper panel shows microscopic images of sphere growth. Bottom panel shows graphic presentation of the average diameter of sphere growth; $\mathrm{n}=3$. (C) Western blotting for CD44, CD133, OCT4 and Nanog in the SCC25 cells transfected with TRIM14expressing plasmids and empty vectors (mock). $\beta$-actin was used as a loading control; $n=3$. (D) Colony formation assay for SCC 25 cells transfected with TRIM14-expressing plasmids and empty vectors (mock). Upper panel shows microscopic images of colony formation. Bottom panel shows graphic presentation of the mean no. of colony formation; $\mathrm{n}=3$. (E) Phase-contrast images of SCC25 cells transfected with TRIM14-expressing plasmids and empty vectors (mock); n=3. (F) Western blotting for E-cadherin and vimentin in the SCC 25 cells transfected with TRIM14-expressing plasmids and empty vectors (mock). $\beta$-actin was used as a loading control; $\mathrm{n}=3$.

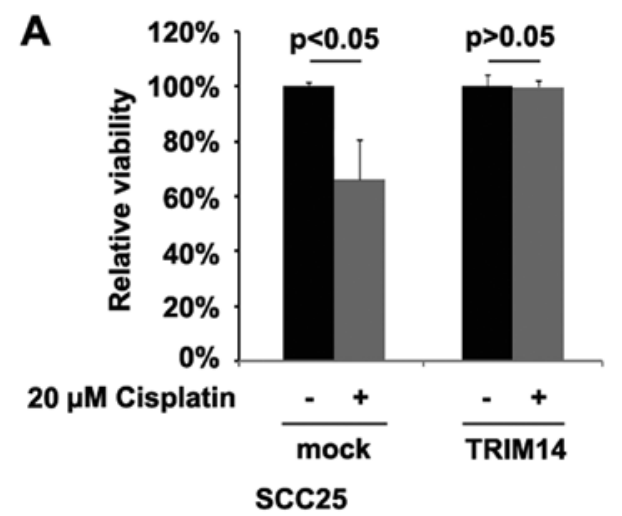

B

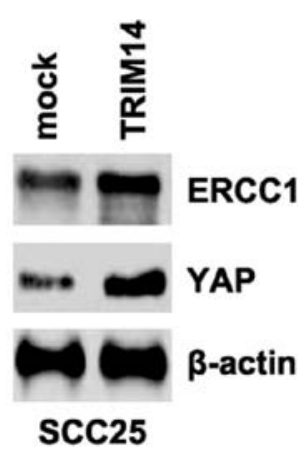

Figure 2. TRIM14 promotes cisplatin-resistance in TSCC. (A) MTT assay for assessement of the cell viability in SCC25 cells. SCC25 cells transfected with the TRIM14-expressing plasmids and empty vectors (mock) were untreated ot treated with cisplatin. (B) Western blotting for ERCC1 and YAP in the SCC25 cells transfected with the TRIM14-expressing plasmids and empty vectors (mock). $\beta$-actin was used as a loading control; $n=3$. TSCC, tongue squamous cell carcinoma. 
A

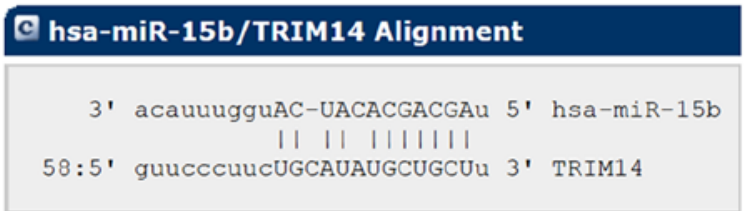

B

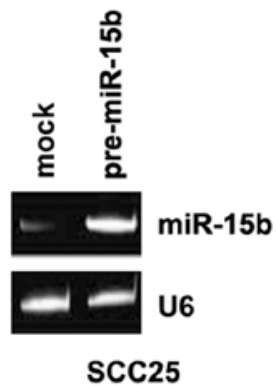

C

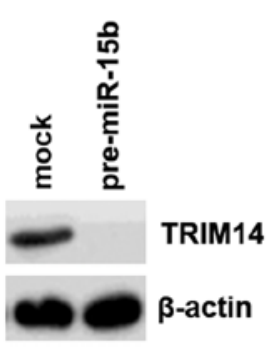

D

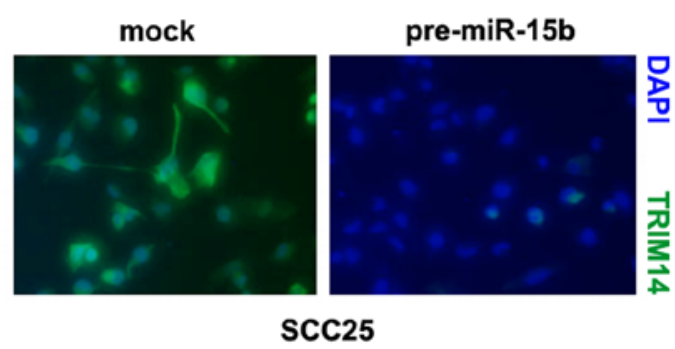

$\mathbf{E}$

$\mathbf{F}$

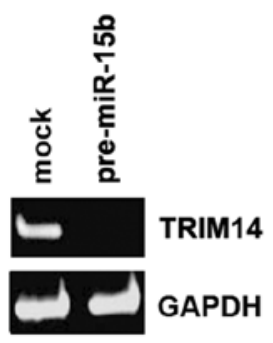

$\operatorname{SCC} 25$

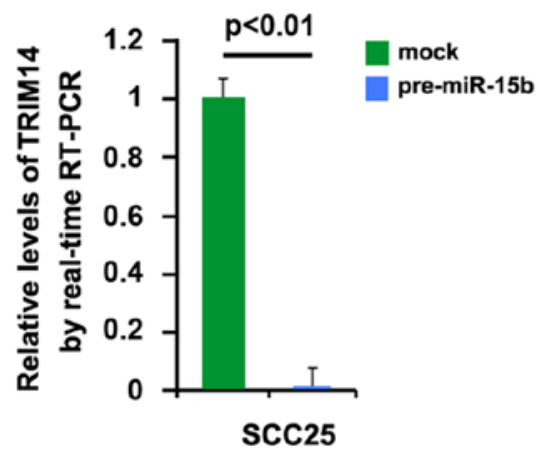

Figure 3. miR-15b degrades TRIM14 in TSCC. (A) Schematic of predicted miR-15b-binding sites in the 3'UTR of TRIM14 mRNA by miRanda. (B) Real-time PCR for miR-15b in the SCC25 cells transfected with pre-miR-15b and control miR. U6 was used as a loading control; $\mathrm{n}=3$. (C) Western blotting for TRIM14 in SCC25 cells transfected with pre-miR-15b and control miR (mock). $\beta$-actin was used as a loading control; $n=3$. (D) Immunofluorescence analyses for TRIM14 in SCC25 cells transfected with pre-miR-15b and control miR (mock); n=3. (E) RT-PCR for TRIM14 in the SCC25 cells transfected with pre-miR-15b and control miR (mock). GAPDH was used as a loading control; n=3. (F) Real-time PCR for TRIM14 in the SCC25 cells transfected with pre-miR-15b and control $\mathrm{miR}$ (mock). GAPDH was used as a loading control; $n=3$. TSCC, tongue squamous cell carcinoma.

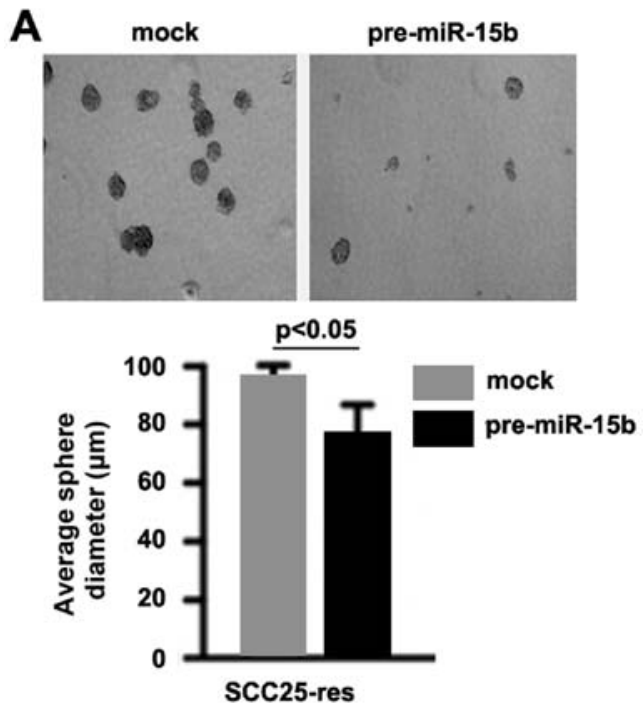

B

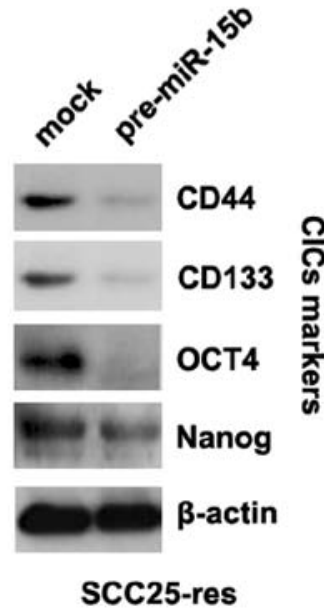

Figure 4. miR-15b induces differentation of cancer-initiating cell (CIC) phenotypes in SCC25-res (cisplatin-resistant) cells. (A) Sphere growth of SCC25 cells transfected with pre-miR-15b and control miR (mock). The data are reported as the average diameter of spheres formed/1,000 seeded cells. Upper panel shows microscopic images of sphere growth. Bottom panel shows graphic presentation of the average diameter of sphere growth; $n=3$. (B) Western blotting for CD44, CD133, OCT4 and Nanog in SCC25 cells transfected with pre-miR-15b and control miR (mock). $\beta$-actin was used as a loading control; $n=3$. 
A
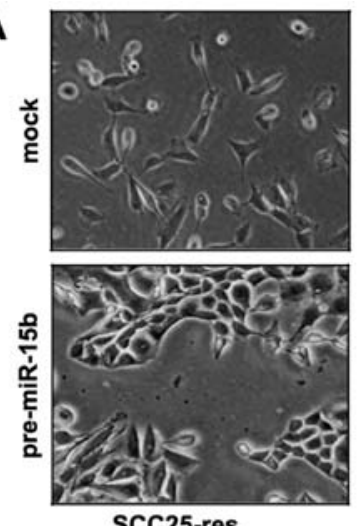

C

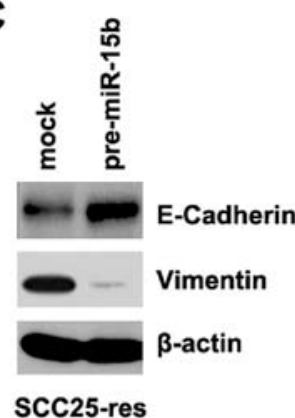

E
B
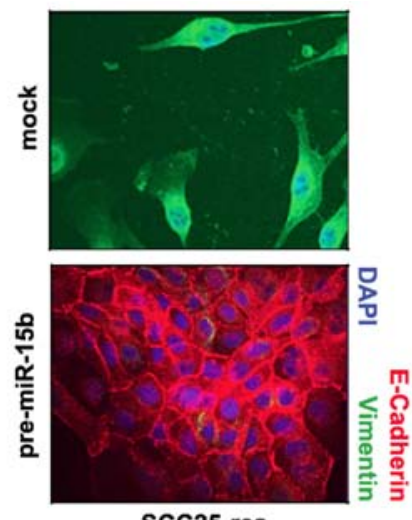

ScC25-res

D

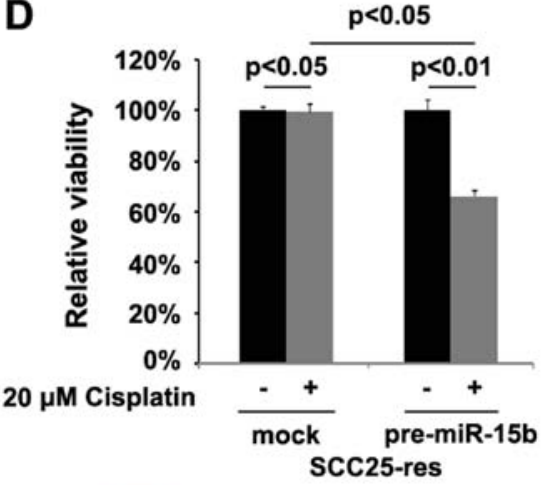

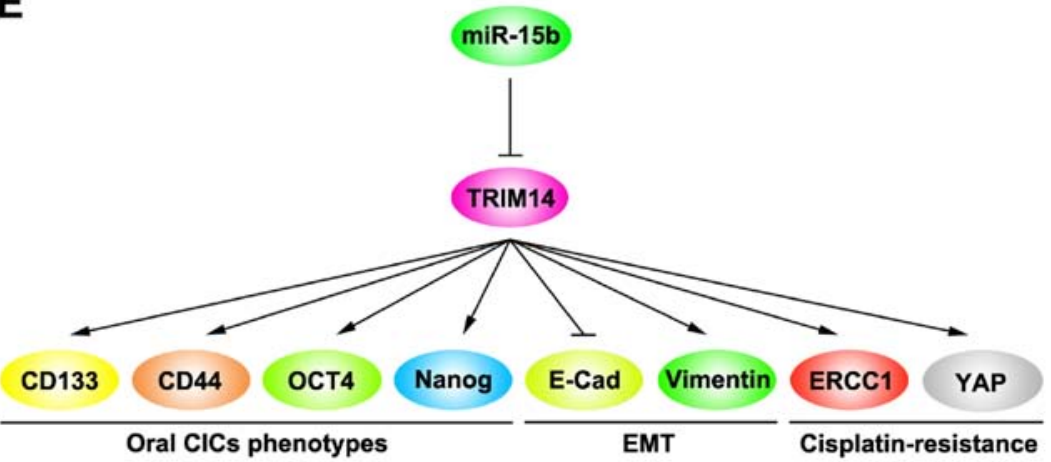

Figure 5. miR-15b induces mesenchymal-epithelial transition (MET) and sensitize SCC25-res cells to cisplatin. (A) Phase-contrast images of SCC25-res cells transfected with pre-miR-15b and control miR (mock); $n=3$. (B) Immunofluorescence analyses for E-cadherin and vimentin in SCC25-res cells transfected with pre-miR-15b and control miR (mock); $n=3$. (C) Western blotting for E-cadherin and vimentin in SCC25-res cells transfected with pre-miR-15b and control miR (mock). $\beta$-actin was used as a loading control; $n=3$. (D) MTT assay for cell viability in the SCC25-res cells. SCC25-res cells transfected with pre-miR-15b and control miR (mock) were untreated or treated with cisplatin. (E) miR-15b induces the differentiation of cancer-initiating cell (CIC) phenotypes and inhibits epithelial-mesenchymal transition (EMT) and cisplatin resistance by regulating TRIM14 in TSCC. TSCC, tongue squamous cell carcinoma.

in locally advanced squamous cell carcinoma of the head and neck $(23,24)$. Our results showed that ERCC1 and YAP protein were upregulated by TRIM14 (Fig. 2B).

miR-15b degrades TRIM14 in TSCC. Having demonstrated that overexpression of TRIM14 induced the formation of CIC phenotypes and EMT, next we studied the mechanisms regulating TRIM14 expression in TSCC. miRs are a class of small non-coding RNAs ( 22 nucleotides) and negatively regulate protein-coding gene expression by targeting mRNA degradation or translation inhibition (11-13).

To further confirm whether TRIM14 could be regulated by miRNA, we used the commonly used prediction algorithm miRanda (http://www.microrna.org/microrna/home.do) to analyze 3'UTR of TRIM14. A dozen miRNAs were identified by the algorithm. However, we were interested in miR-15b, since it is one of the most significantly downregulated miRNAs in SCC25-res cells and its restoration effectively reversed the phenotype of EMT in SCC25-res cells, and sensitized them to chemotherapy (25).

Target sites on 3'UTR of TRIM14 are shown in Fig. 3A. We reasoned that miR-15b downregulates TRIM14 expression by targeting its 3'UTR in TSCC. Downregulation of miR-15b can contribute to upregulation of TRIM14 and cisplatin resistance in TSCC. In an attempt to identify the role of miR-15b in regulating TRIM14 expression in TSCC, we transfected SCC25 cells with pre-miR-15b and control miR. After transfection, miR-15b expression was detected by real-time PCR and the results showed that miR-15b was significantly increased by pre-miR-15b in the cells (Fig. 3B). 
To confirm the reason, we performed western blotting to detect TRIM14 expression in the SCC25 cells transfected with pre-miR-15b and control miR. The results showed that TRIM14 protein was significantly inhibited by miR-15b (Fig. 3C). We next performed immunofluorescence analyses in the SCC25 cells transfected with pre-miR-15b and control miR. The results showed that TRIM14 protein was evidently inhibited in the cells transfected with pre-miR-15b (Fig. 3D). To identify whether miR-15b can degrade TRIM14 mRNA, we performed RT-PCR and real-time PCR and we found that TRIM14 mRNA was degraded by miR-15b (Fig. 3E and F).

miR-15b inhibits CIC phenotypes in the SCC25-res cells. In order to ascertain whether miR-15b affects CIC traits in the SCC25-res cells, we performed sphere forming assay to assess the capacity of CIC or CIC-like self renewal in the SCC25-res cells. Sphere forming assay showed that miR$15 \mathrm{~b}$-overexpressing cells formed much smaller spheres after 14 days of culture as compared with control cells, indicating markedly decreased CIC traits by pre-miR-15b (Fig. 4A). To identify whether TRIM14 can regulate CD44, CD133, OCT4 and Nanog protein expression, we performed western blotting in SCC25-res cells transfected with pre-miR-15b and control miR. The results showed that CD44, CD133, OCT4 and Nanog protein were downregulated in the SCC25-res cells transfected with pre-miR-15b (Fig. 4B).

miR-15b induces MET and sensitizes SCC25-res cells to cisplatin. Cell morphology demonstrated that overexpression of miR-15b induced MET phenotypes in SCC25-res cells (Fig. 5A). In order to detect whether miR-15b can affect E-cadherin (epithelial marker) and vimentin (mesenchymal marker) protein, we performed immunofluorescence analyses in the SCC25-res cells transfected with pre-miR$15 \mathrm{~b}$ and control $\mathrm{miR}$. We found that restoration of $\mathrm{miR}-15 \mathrm{~b}$ promoted E-cadherin and inhibited vimentin in the SCC25-res cells (Fig. 5B). We also performed western blotting to detect E-cadherin and vimentin protein in the SCC25-res cells transfected with pre-miR-15b and control miR. We found that E-cadherin protein was upregulated and vimentin was downregulated by miR-15b in the SCC25-res cells (Fig. 5C).

To study the role of miR-15b in cisplatin resistance, we performed MTT assay in the SCC25-res cells. The results showed that restoration of miR-15b sensitized SCC25-res cells to cisplatin (Fig. 5D).

\section{Discussion}

miR-15b is the one of most significantly downregulated microRNAs (miRNAs/miRs) in cisplatin-resistant SCC25 (SCC25-res) cells and its restoration effectively reversed EMT in the SCC25-res cells, and sensitized them to chemotherapy, while inhibition of miR-15b in the sensitive lines induced EMT and conferred chemoresistance (25). Moreover, EMT cells can have cancer-initiating cell-like features and CICs exhibit a mesenchymal phenotype under most circumstances. Consequently, malignant tumors tend to relapse after surgical resection. This character is believed to be largely attributable to the stem cell-like properties of a fraction of cells (26). In the present study, we found that miR-15b may play an important role in the formation of CICs and MET of TSCC and in the regulation of cisplatin (cisplatin is widely used to treat oral squamous cell carcinoma, however, many patients exhibit acquired drug resistance). These findings provide novel insights into the potential role of miR-15b deregulation in promoting the formation of CICs and conferring chemoresistance in TSCC (Fig. 5E).

Ectopic expression of TRIM14 in TSCC cells was found to promote proliferation, angiogenesis and increased resistance to cisplatin-induced apoptosis in TSCC cells in vitro. Furthermore, TRIM14 overexpression significantly promoted the tumorigenicity of TSCC cells in vivo whereas silencing of endogenous TRIM14 caused an opposite outcome (9). In line with this report, we showed that overexpression of TRIM14 induced the formation of CIC traits and EMT (Fig. 5E). The results further confirmed the idea that CICs exhibiting a mesenchymal phenotype frequently can promote chemotherapy resistance in cancer. Contrary to miR-15b, TRIM14 is markedly upregulated in TSCC cell lines and clinical tissues $(9,25)$. Restoration of miR-15b to inhibit TRIM14 may represent a novel therapeutic target for the treatment of TSCC.

Low expression of ERCC1 is an independent predictor for prolonged survival (23). Moreover, it has been proposed that ERCC1 expression may be a useful predictive marker of locally advanced squamous cell carcinoma of the head and neck in patients treated with cisplatin-based concurrent chemoradiotherapy (23). We demonstrated that TRIM14 overexpression significantly promoted ERCC1 expression in the TSCC cells. Deregulation of the miR-15b/TRIM14/ERCC1 axis may play an important role in the progression of TSCC. YAP overexpression correlates with epithelial-mesenchymal transition and nodal metastasis, resulting in cisplatin-resistance. Thus, targeting YAP could be a new therapeutic strategy for the treatment of patients with oral squamous cell carcinoma that are resistant to cisplatin (24). We found that TRIM14 significantly upregulated YAP protein level in the SCC25 cells (Fig. 5E). Deregulation of the miR-15b/TRIM14/YAP axis may promote the formation of cisplatin-resistant TSCC.

\section{Acknowledgements}

The present study was supported by the Shandong Province Science and Technology Research Project (2013G0021812).

\section{References}

1. Jemal A, Siegel R, Ward E, Murray T, Xu J and Thun MJ: Cancer statistics, 2007. CA Cancer J Clin 57: 43-66, 2007.

2. Ferlay J, Shin HR, Bray F, Forman D, Mathers C and Parkin DM: Estimates of worldwide burden of cancer in 2008: GLOBOCAN 2008. Int J Cancer 127: 2893-2917, 2010.

3. Argiris A, Karamouzis MV, Raben D and Ferris RL: Head and neck cancer. Lancet 371: 1695-1709, 2008.

4. Rusthoven K, Ballonoff A, Raben D and Chen C: Poor prognosis in patients with stage I and II oral tongue squamous cell carcinoma. Cancer 112: 345-351, 2008.

5. Brennan S, Corry J, Kleid S, Porceddu S, Yuen K, Rischin D and Peters LJ: Prospective trial to evaluate staged neck dissection or elective neck radiotherapy in patients with CT-staged T1-2 N0 squamous cell carcinoma of the oral tongue. Head Neck 32: 191-198, 2010.

6. Dean M, Fojo T and Bates S: Tumour stem cells and drug resistance. Nat Rev Cancer 5: 275-284, 2005.

7. Zhang Q, Shi S, Yen Y, Brown J, Ta JQ and Le AD: A subpopulation of $\mathrm{CD}_{133^{+}}$cancer stem-like cells characterized in human oral squamous cell carcinoma confer resistance to chemotherapy. Cancer Lett 289: 151-160, 2010. 
8. Meroni G and Diez-Roux G: TRIM/RBCC, a novel class of 'single protein RING finger' E3 ubiquitin ligases. BioEssays 27: 1147-1157, 2005.

9. Su X, Wang J, Chen W, Li Z, Fu X and Yang A: Overexpression of TRIM14 promotes tongue squamous cell carcinoma aggressiveness by activating the NF- $\kappa \mathrm{B}$ signaling pathway. Oncotarget 7: 9939-9950, 2016.

10. Bartel DP: MicroRNAs: Genomics, biogenesis, mechanism, and function. Cell 116: 281-297, 2004.

11. Lee RC, Feinbaum RL and Ambros V: The C. elegans heterochronic gene lin-4 encodes small RNAs with antisense complementarity to lin-14. Cell 75: 843-854, 1993.

12. Pasquinelli AE, Reinhart BJ, Slack F, Martindale MQ, Kuroda MI, Maller B, Hayward DC, Ball EE, Degnan B, Müller P, et al: Conservation of the sequence and temporal expression of let-7 heterochronic regulatory RNA. Nature 408: 86-89, 2000.

13. Reinhart BJ, Slack FJ, Basson M, Pasquinelli AE, Bettinger JC Rougvie AE, Horvitz HR and Ruvkun G: The 21-nucleotide let-7 RNA regulates developmental timing in Caenorhabditis elegans. Nature 403: 901-906, 2000.

14. Lewis BP, Burge CB and Bartel DP: Conserved seed pairing, often flanked by adenosines, indicates that thousands of human genes are microRNA targets. Cell 120: 15-20, 2005.

15. Farh KK, Grimson A, Jan C, Lewis BP, Johnston WK, Lim LP, Burge CB and Bartel DP: The widespread impact of mammalian MicroRNAs on mRNA repression and evolution. Science 310 : 1817-1821, 2005 .

16. Duz MB, Karatas OF, Guzel E, Turgut NF, Yilmaz M, Creighton CJ and Ozen M: Identification of miR-139-5p as a saliva biomarker for tongue squamous cell carcinoma: A pilot study. Cell Oncol 39: 187-193, 2016.

17. Kong D, Banerjee S, Ahmad A, Li Y, Wang Z, Sethi S and Sarkar FH: Epithelial to mesenchymal transition is mechanistically linked with stem cell signatures in prostate cancer cells. PLoS One 5: e12445, 2010
18. Ren ZG, Dong SX, Han P and Qi J: miR-203 promotes proliferation, migration and invasion by degrading SIK1 in pancreatic cancer. Oncol Rep 35: 1365-1374, 2016.

19. Park SY, Lee HE, Li H, Shipitsin M, Gelman R and Polyak K: Heterogeneity for stem cell-related markers according to tumor subtype and histologic stage in breast cancer. Clin Cancer Res 16: 876-887, 2010.

20. Mansour SF and Atwa MM: Clinicopathological significance of CD133 and ALDH1 cancer stem cell marker expression in invasive ductal breast carcinoma. Asian Pac J Cancer Prev 16 7491-7496, 2015

21. Tsai LL, Hu FW, Lee SS, Yu CH, Yu CC and Chang YC: Oct4 mediates tumor initiating properties in oral squamous cell carcinomas through the regulation of epithelial-mesenchymal transition. PLoS One 9: e87207, 2014.

22. Chiou SH, Yu CC, Huang CY, Lin SC, Liu CJ, Tsai TH, Chou SH, Chien CS, Ku HH and Lo JF: Positive correlations of Oct-4 and Nanog in oral cancer stem-like cells and high-grade oral squamous cell carcinoma. Cancer Res 14: 4085-4095, 2008

23. Jun HJ, Ahn MJ, Kim HS, Yi SY, Han J, Lee SK, Ahn YC, Jeong HS, Son YI, Baek JH, et al: ERCC1 expression as a predictive marker of squamous cell carcinoma of the head and neck treated with cisplatin-based concurrent chemoradiation. Br J Cancer 99: 167-172, 2008.

24. Yoshikawa K, Noguchi K, Nakano Y, Yamamura M, Takaoka K, Hashimoto-Tamaoki $\mathrm{T}$ and Kishimoto H: The Hippo pathway transcriptional co-activator, YAP, confers resistance to cisplatin in human oral squamous cell carcinoma. Int J Oncol 46: 2364-2370, 2015.

25. Sun L, Yao Y, Liu B, Lin Z, Lin L, Yang M, Zhang W, Chen W, Pan C, Liu Q, et al: MiR-200b and miR-15b regulate chemotherapyinduced epithelial-mesenchymal transition in human tongue cancer cells by targeting BMI1. Oncogene 31: 432-445, 2012.

26. Sanai N, Alvarez-Buylla A and Berger MS: Neural stem cells and the origin of gliomas. N Engl J Med 353: 811-822, 2005. 